\title{
Primary Retrovesical Hydatid Cyst: Two Cases Report with Review
}

\author{
Snigdha Goyal ${ }^{*}$, Isha Saini' ${ }^{2}$, Sunder Goyal ${ }^{3}$ \\ ${ }^{1}$ Department of Pathology, Dr. RML Postgraduate Institute of Medical Sciences, New Delhi, India \\ ${ }^{2}$ Kalpna Chawla Medical College, Karnal, Haryana, India \\ ${ }^{3}$ Department of Surgery, Kaplna Chawla Medical College, Karnal, Haryana, India \\ Email: drgoyal4@gmail.com, ishasdoc@gmail.com, goyal.sunder@yahoo.in
}

Received 26 June 2015; accepted 13 July 2015; published 21 July 2015

Copyright (C) 2015 by authors and OALib.

This work is licensed under the Creative Commons Attribution International License (CC BY).

http://creativecommons.org/licenses/by/4.0/

(c) (i) Open Access

\begin{abstract}
Hydatid cyst is common in the liver and lung but extremely rare in pelvic cavity. Pelvic hydatid cyst may present with pressure symptoms on ureter or urinary bladder and may result in bilateral hydronephrosis or retention of urine. Bilateral hydroureteronephrosis is one of the rare presentations of pelvic hydatid disease. Hereby, we report two cases of primary pelvic hydatid cyst: one was with bilateral hydronephrosis with nonfunctioning right kidney and another was asymptomatic.
\end{abstract}

\section{Keywords}

\section{Pelvic Hydatid Cyst, Bilateral Hydronephrosis, Liver}

Subject Areas: Surgery \& Surgical Specialties

\section{Introduction}

Cystic echinococcosis is an endemic problem in Middle East and Asia. Cystic echinococcosis mostly affects lung, liver and spleen but it may also occur rarely in unusual sites as bone, muscle, brain, adrenal and pelvis [1]. Primary hydatid disease of the pelvis $(0.75 \%-2.25 \%)$ is a very rare disease [2]-[5]. According to another clinical-epidemiological study which was carried out among 117 cases of hydatid disease in central India, only 1 case of pelvic hydatidotosis was reported. However, in endemic country like Libya, only 14 cases of pelvic hydatid cyst were reported in one institution between 1971 and 1979 [6]. Incidence of retrovesical hydatid cyst is about $0.1 \%-0.5 \%$ of hydatid cysts disease [7]. Due to its location in a fixed cavity, it presents with pressure effects on adjacent organs such as urinary bladder, ureter or rectum. Urinary symptoms may be retention of urine, frequency of micturition or as obstructive uropathy and renal failure [5] [8] [9]. Rarely, pelvic hydatid cyst can result in neurological symptoms in lower extremities due to mass effect of pelvis [10]. Hereby, we report two

\footnotetext{
"Corresponding author.
} 
cases of primary pelvic hydatid cyst: one was with bilateral hydronephrosis with nonfunctioning right kidney and another was asymptomatic.

\section{Material and Methods}

A study was carried out in a medical college about the incidence of hydatid disease during Jan. 2012 to June 2014. A total 20 cases of hydatid disease were encountered. 12 cases were of liver hydatid disease, 4 cases were of lung hydatid cyst, one with spleen hydatid and only 2 cases were of pelvic hydatid disease.

We describe two cases of pelvic hydatid cysts, one with bilateral hydronephrosis due to local compression of ureter and the other one was asymptomatic. The radiology and pathology confirmed the diagnosis in the first case and only radiological findings confirmed the second. Both patients are now asymptomatic in follow up period.

\section{1. $1^{\text {st }}$ Case}

A 50 year male presented with retention of urine for the last one day. He gave past history of difficulty in micturition for last five years along with pain in right loin for last six months. Examination revealed a firm, smooth, non mobile and non tender mass in suprapubic region along with a ballotable mass in right lumbar region. On per rectal examination, a smooth cystic mass was felt anteriorly in the rectovesical pouch. Serum creatinine, urea and electrolytes, liver function test and urine analysis were normal. Ultrasound examination revealed a cystic mass in pelvis suggestive of a hydatid cyst with bilateral hydronephrosis. There was no lesion in the liver. Computerized tomography (CT) scan revealed a pelvic hydatid cyst with bilateral hydronephrosis with non functioning right kidney (Figure 1, Figure 2). Indirect haemagglutination test for hydatid disease was negative.

Bilateral percutaneous nephrostomy was done to relieve the obstruction as we failed to do bilateral ureteric stenting. Right kidney was nonfunctional and left kidney functioned well. Preoperatively patient was put on albendazole $(15 \mathrm{mg} / \mathrm{kg} / \mathrm{day})$ for 4 weeks. Exploratory laparotomy was done after 4 weeks. A cyst was found in rectovesical pouch, which had dense adhesions with urinary bladder, pelvic part of ureters, left iliac vessels and rectum posteriorly (Figure 3). Normal saline (3\%) was used as scolicidal agent. Whole of cyst could not be removed due to dense adhesions. Marsupialization was done and cyst contents (daughter cysts) were evacuated (Figure 4, Figure 5). Omentoplasty was performed to obliterate the residual cavity after washing the cavity with povidine iodine (5\%). Right nephrectomy was done in addition as kidney was non-functioning. Patient was put on albendazole in postoperative period. Patient is asymptomatic for the last one year in follow up.

\section{2. $2^{\text {nd }}$ Case}

The second case of pelvic hydatid disease was asymptomatic, and was discovered by chance while examining a 59-year-old man for benign prostatic enlargement. Because he was asymptomatic, patient was kept under observation and was put on albendazole. He is asymptomatic in follow up period of six months.

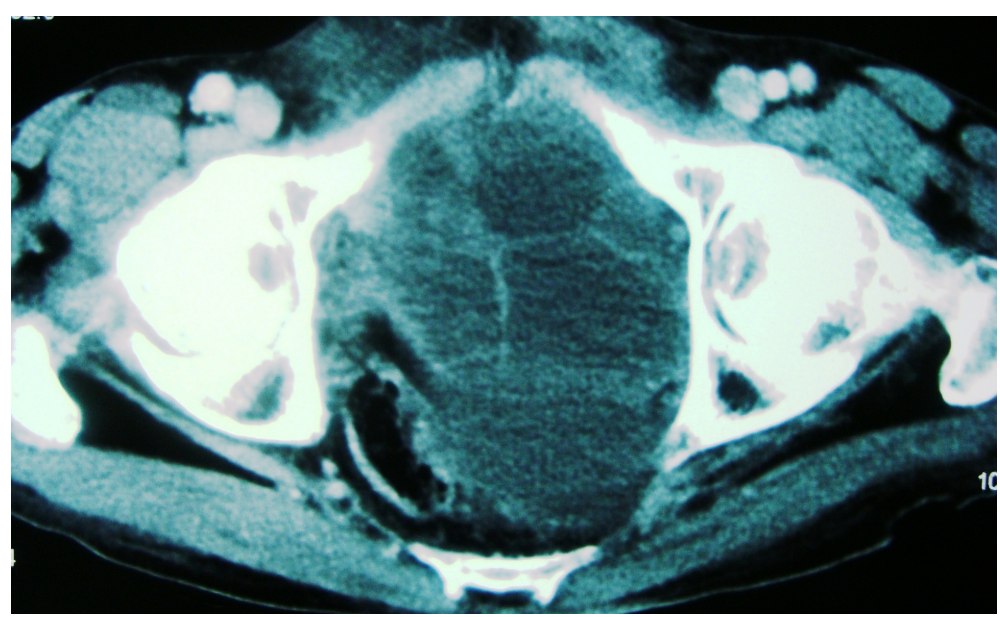

Figure 1. CECT of $1^{\text {st }}$ case showing pelvic hydatid cyst. 


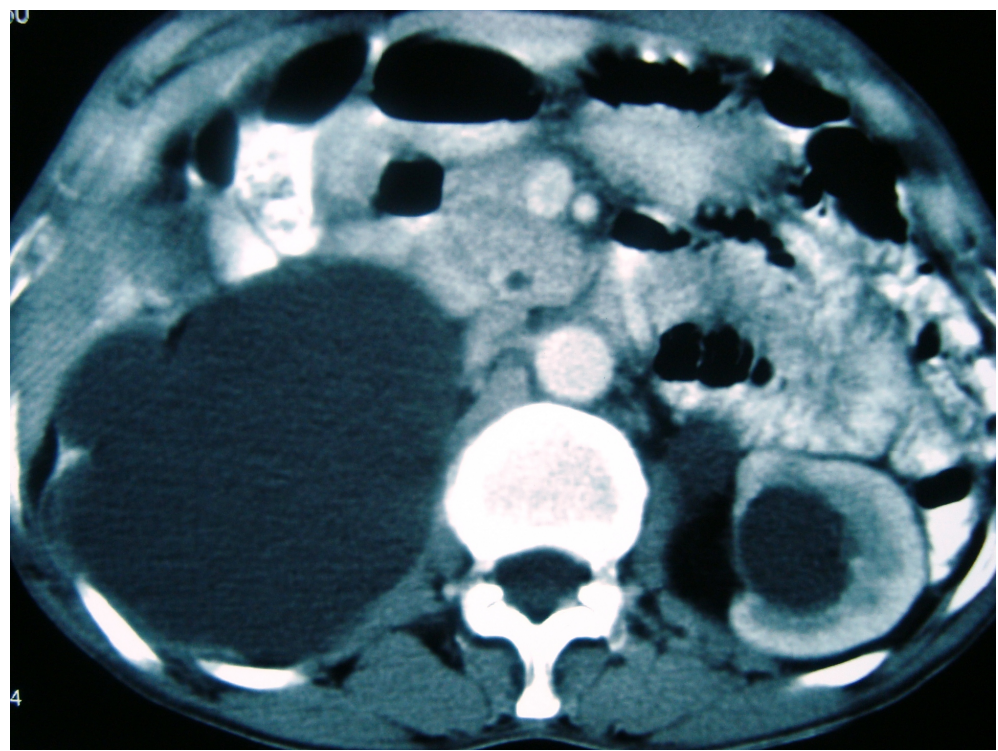

Figure 2. Showing bilateral hydronephrosis.

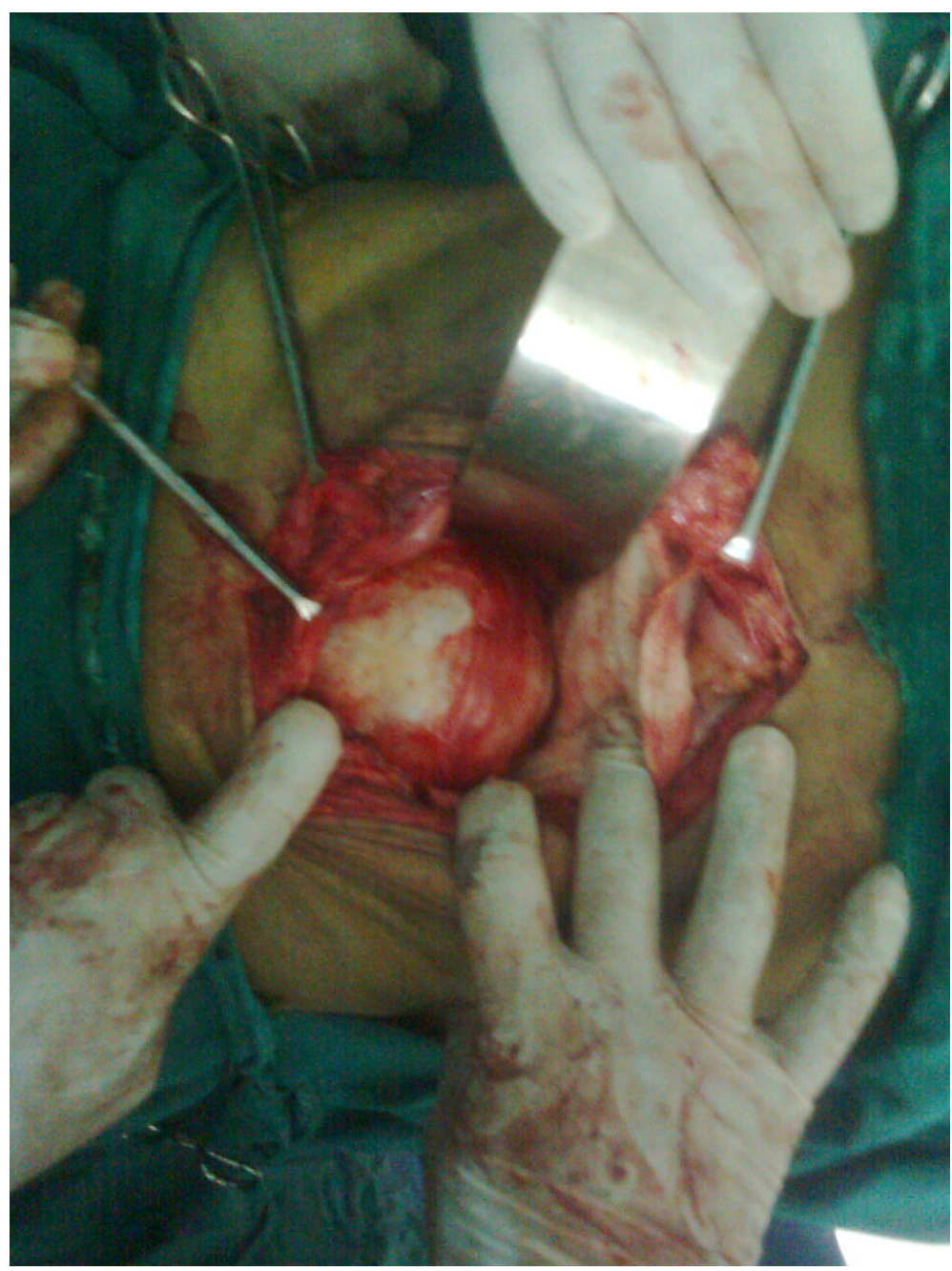

Figure 3. Showing operative procedure. 


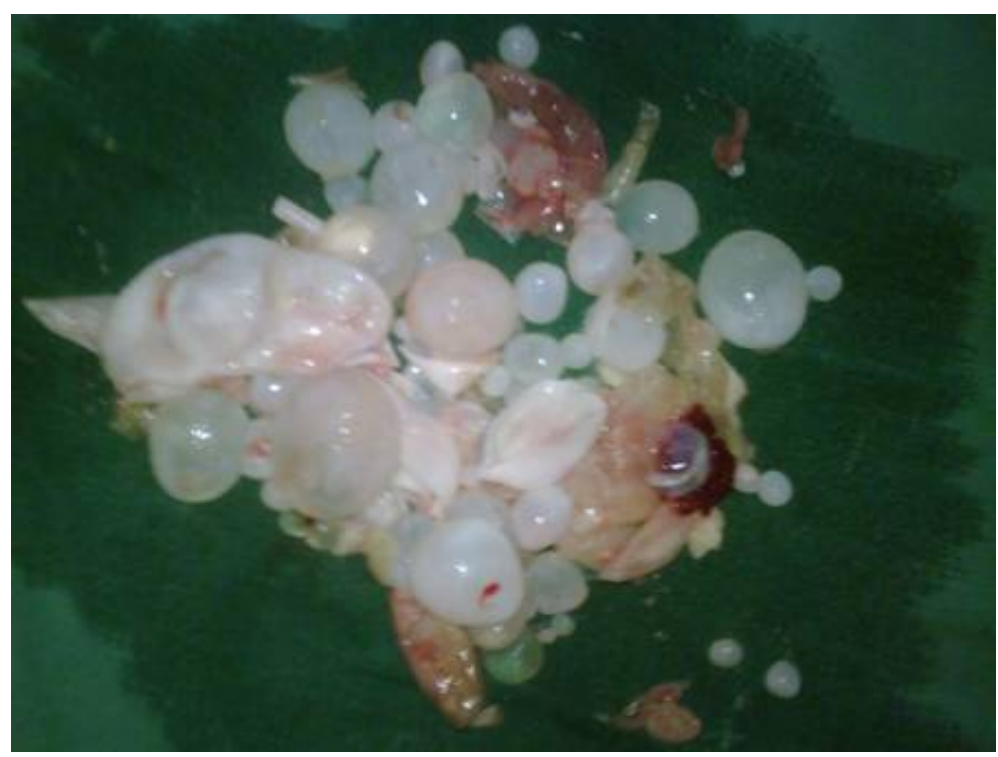

Figure 4. Daughter cysts removed from pelvic cavity hydatid cyst.

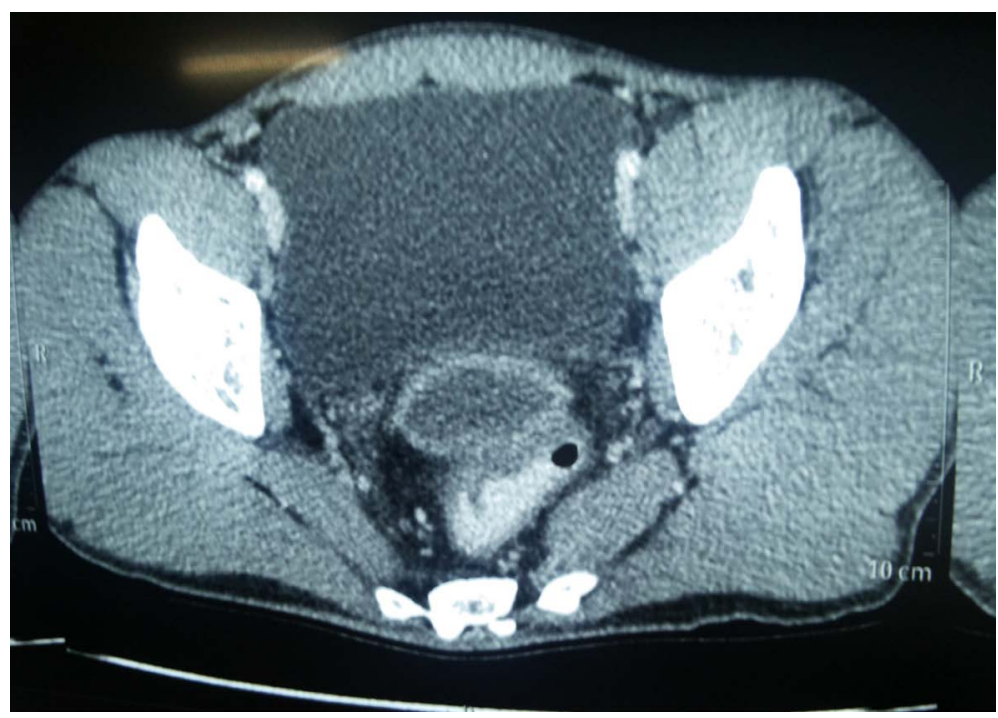

Figure 5. CECT of $2^{\text {nd }}$ case showing retrovesical pelvic hydatid cyst.

\section{Discussion}

Human being gets infestation of echinococcus by swallowing the ova accidentally. In human being infection is usually acquired in childhood and it takes 5 to 20 yrs to develop symptoms. Cysts are found in liver (55\% to $60 \%)$, lung (20\% to $30 \%)$, kidney (2.5\%), heart (2.5\%), bones (2\%), muscle $(1 \%)$, brain $(0.5 \%)$, spleen $(1.5 \%)$ and other rare sites are omentum, ovaries, parametrium, pelvis, thyroid, orbit or retro peritoneum [8] [11].

Hydatid cyst located in the peritoneal cavity or pelvis is usually secondary to spontaneous rupture from a primary liver focus or surgical inoculation. Primary pelvic hydatid cyst is extremely rare with an incidence of about $0.2 \%$ to $2.25 \%$ (with retrovesical location being even rarer). Pelvic localization may be considered primary if no other site is found to be affected (as in our cases).

Connective tissue beneath the pelvic peritoneum is the usual site of origin of pelvic hydatid disease. From here it may spread to uterus, ovaries, fallopian tubes, urinary bladder and or to rectum after contact. Other hypothesis of primary pelvic hydatid disease may be that retroperitoneal inoculation occur by penetration of rectum by larva and transmission to chest with lymphatic spread consequently [1]. 
Preoperative diagnosis of primary retroperitoneal hydatid cysts is not easy and these can mimic a pelvic malignant tumor with pressure effects on ureter leading to bilateral hydronephrosis (as in our one case). These presentations are more atypical in children [1]. The growth rate of liver hydatid cyst is about $1-2 \mathrm{~cm} /$ year but few guess that growth rate is $5-10 \mathrm{~cm}$ in size within the first year. But estimation of growth rate of retroperitoneal cysts is not clear so far [12].

Retrovesical hydatid cysts may have wide-ranging and non-specific symptoms. The most common presenting symptoms may be a palpable mass followed by flank pain, frequency, urinary retention and pain on micturition [9]. Pelvic hydatid disease can present with urine retention of urine, bilateral hydronephrosis and rarely with obstructive uropathy, most often in male patients [5] [13]. Less than 15\% of retrovesical hydatid cysts can create fistula with the bladder resulting in hydatiduria which is a pathognomonic sign of vesicular cystic fistula [14].

Hydatid cyst should always be considered in differential diagnosis of abdomeno-pelvic masses like rectal duplication cyst, rectosigmoid neoplasm, posterior bladder diverticulum, cyst of the seminal vesicle, and hydronephrosis in a pelvic kidney, large ectopic ureterocoele in male patients and mullerian remnant, hydrosalpinx, pseudomyxoma peritonei, tubal pregnancy in female patients in endemic regions.

Various serological tests like immunoelectrophoresis, enzyme linked immunosorbent assay (ELISA), latex agglutination and indirect haemagglutination (IHA) are carried out for the diagnosis, screening and postoperative follow up for recurrence but these tests are often negative because the capsule isolate the parasite from the host's immune system. Serologic tests may be specific and sensitive for liver hydatid disease but these tests can be negative for other organs hydatid disease [12].

Ultrasonography and computed tomography (CT) are both excellent imaging modalities for the detection of hydatid cysts. Ultrasonography is the important diagnostic tool in cases of hydatid cysts and sensitivity range from $93 \%$ to $98 \%$. On ultrasound, the double line wall sign is highly suggestive of hydatid cyst. CT is also useful to confirm the diagnosis and sensitivity ranges from $90 \%$ to $98 \%$ [9].

Preoperative albendazole treatment reduces the size and the viability of the cysts thus decreases chances of recurrence. The duration of the preoperative treatment varies from 48 hrs (minimum) to 4 weeks. Correct preoperative diagnosis is very important as if scolicidal precautions are not taken during surgery, dissemination and seedling cannot be avoided. The surgery is treatment of choice and total cyst excision (total pericystectomy) without spillage and contamination of the field is done. Due to location within the narrow confines of the pelvis and due to dense adhesions to surrounding structures, it is very difficult to do total removal of pelvic hydatid cyst. Partial pericystectomy may have to be done in situations where separation from neighbouring structures is not possible. Omentoplasty is done to fill the dead space (as in our case). Kumar et al. [15] described two cases of retrovesical hydatid cysts which were managed laparoscopically. Cyst aspiration, instillation of 3\% saline and suction of daughter cysts was done with the help of laparoscope. The da Vinci ${ }^{\circledR}$ surgical robotic system can also be used for the surgical excision of retrovesical hydatid cyst as robotic system enhances magnification, provide 3-D vision with endowrist technology.

In our 1st case, cyst was occupying whole of the pelvis with pressure on pelvic part of both ureter resulting in bilateral hydronephrosis with nonfunctioning right kidney. Surgery was conducted in $1^{\text {st }}$ case and $2^{\text {nd }}$ case was kept under observation and both patients were given a course of albendazole. The pathology confirmed the diagnosis in the first case and radiological imaging modality confirmed the diagnosis in the second case. Both patients are now asymptomatic with follow up to six months.

\section{Conclusion}

Pelvic echinococcosis is rare, with an incidence of $0.2 \%-2.25 \%$. Retrovesical location of cyst is even rarer. In endemic countries, it should always be in the differential diagnosis of any tumor like growing mass of pelvis even in the absence of accompanying involvement of liver or lung.

\section{References}

[1] Malaki, M. and Jamshidi, M. (2013) A Pelvic Mass with Obstructive Uropathy in a 4-Year Old Boy. Annals of Tropical Medicine and Public Health, 6, 306-308. http://dx.doi.org/10.4103/1755-6783.120990

[2] Halefoglu, A.M. and Yasar, A. (2007) Huge Retrovesical Hydatid Cyst with Pelvic Localization as the Primary Site: A Case Report. Acta Radiologica, 48, 918-920. http://dx.doi.org/10.1080/02841850701422138

[3] Soumakis, K., Altanis, S. and Katsiva, V. (2007) Primary Pelvic Hydatid Cyst. Journal of Obstetrics and Gynaecology, 
27, 745-746. http://dx.doi.org/10.1080/01443610701629247

[4] Goel, A., Tiwari, P., Sharma, P.K., Kumar, S. and Kundu, A.K. (2013) Extragenitourinary Retroperitoneal Primary Hydatid Cyst: A Rare Cause of Bilateral Lower Ureteric Obstruction and Unilateral Limb Edema. Saudi Journal of Kidney Diseases and Transplantation, 24, 991-994. http://dx.doi.org/10.4103/1319-2442.118092

[5] Emir, L., Karabulut, A., Balci, U., Germiyanoglu, C. and Erol, D. (2000) An Unusual Cause of Urinary Retention: A Primary Retrovesical Echinococcal Cyst. Urology, 56, 856. http://dx.doi.org/10.1016/S0090-4295(00)00759-7

[6] Bhattacharya, A., Saha, R., Mitra, S. and Nayak, P. (2013) Primary Hydatid Cyst of Broad Ligament. Tropical Parasitology, 3, 155-157. http://dx.doi.org/10.4103/2229-5070.122148

[7] Rodriguez, L.S. (2013) Primary Retrovesical Hydatidosis as a Cause of Chronic Kidney Disease. Nefrologia, 33, 285286.

[8] Seenu, V., Mishra, M.C., Tiwari, S.C., Jain, R. and Chanrashekhar, C. (1994) Primary Pelvic Hydatid Cyst Presenting with Obstructing Uropathy and Renal Failure. Postgraduate Medical Journal, 70, 930-932. http://dx.doi.org/10.1136/pgmj.70.830.930

[9] Saadeh, R.J. and Mohamed, A.R. (2010) Urinary Retention: Unusual Presentation of Hydatid Cyst: Case Report \& Literature Review. The Internet Journal of Surgery, 27.

[10] Maddah, G., Shabanang, H. and Noghabi, R.S. (2013) Pelvic Hydatid Cyst with Uncommon Sciatalgia Manifestation: A Case Report. Tehran University Medical Journal (TUMJ), 71, 541-545.

[11] Sekar, N., Madhavan, K.K., Yadav, R.V. and Katariya, R.N. (1982) Primary Retroperitoneal Hydatid Cyst: Case Report of 3 Cases and Review of Literature. Postgraduate Medical Journal, 28, 112-148.

[12] Macpherson, C.N. and Milner, R. (2003) Performance Characteristics and Quality Control of Community Based Ultrasound Surveys for Cystic and Alveolar Echinococcosis. Acta Tropica, 85, 203-209. http://dx.doi.org/10.1016/S0001-706X(02)00224-3

[13] Kilicarslan, H., Gokce, G., Kaya, S., Atalar, M., Ayan, S., Kaya, K. and Gultekin, Y. (2003) A Case of Hydatid Disease: Urinary Retention Due to an Isolated Retrovesical Hydatid Cyst in a Boy. Scandinavian Journal of Infectious Diseases, 35, 408-410. http://dx.doi.org/10.1080/00365540310011001

[14] Touiti, D., Ameur, A., Chouhou, K., Alkandry, S., Oukheira, H. and Borki, K. (2001) Hydatid Cyst of the Douglas's Cul-De-Sac with Fistula to the Bladder. Report of 2 Cases. Annales d'Urologie (Paris), 35, 216-219. http://dx.doi.org/10.1016/S0003-4401(01)00029-8

[15] Kumar, S., Pandya, S., Agrawal, S. and Lal, A. (2008) Laparoscopic Management of Genitourinary Hydatid Cyst Disease. Journal of Endourology, 22, 1709-1713. http://dx.doi.org/10.1089/end.2008.0128 